QUARTERLY OF APPLIED MATHEMATICS

VOLUME LXVII, NUMBER 2

JUNE 2009, PAGES 311-326

S 0033-569X(09)01112-8

Article electronically published on March 20, 2009

\title{
ASYMPTOTICS OF RESONANCES IN A THERMOELASTIC MODEL WITH LIGHT LOCAL MASS PERTURBATIONS
}

BY

NATALIA BABYCH (Department of Mathematical Sciences, University of Bath, Bath BA2 7AY, United Kingdom)

AND

JOHANNES ZIMMER (Department of Mathematical Sciences, University of Bath, Bath BA2 7AY, United Kingdom)

Abstract. The limit behaviour of a linear one-dimensional thermoelastic system with local mass perturbations is studied. The mass density is supposed to be nearly homogeneous everywhere except in an $\varepsilon$-vicinity of a given point, where it is of order $\varepsilon^{-m}$, with $m \in \mathbb{R}$. The resonance vibrations of the string are investigated as $\varepsilon \rightarrow 0$. An important ingredient of the analysis is the construction of an operator in a space of higher regularity such that its spectrum coincides with that of the classical operator in linearised thermoelasticity, with a correspondence of generalised eigenspaces. The convergence of eigenvalues and eigenprojectors is established along with error bounds for two classes of relatively light mass perturbations, $m<1$ and $m=1$, which exhibit contrasting limit behaviour.

1. Statement of the problem. We consider resonance vibrations of a finite string modelled in the framework of linearised, one-dimensional thermo-elasticity. The evolution of the displacement $u=u(x, t)$ and the relative temperature $\theta=\theta(x, t)$ is governed by the system of differential equations [9, 8, 5]

$$
\begin{gathered}
\rho_{\varepsilon}(x) \frac{\partial^{2} u}{\partial t^{2}}-\frac{\partial}{\partial x}\left(\alpha(x) \frac{\partial u}{\partial x}\right)+\frac{\partial}{\partial x} \beta(x) \theta=f(x, t), \\
k(x) \frac{\partial \theta}{\partial t}+\beta(x) \frac{\partial^{2} u}{\partial x \partial t}-\frac{\partial}{\partial x}\left(\varkappa(x) \frac{\partial \theta}{\partial x}\right)=\phi(x, t),
\end{gathered}
$$

where $\rho_{\varepsilon}$ is the mass density of the string, $\alpha$ is a stiffness coefficient, $k$ is the specific heat, $\varkappa$ denotes the thermal conductivity coefficient, and $\beta$ is a coupling coefficient; $f$ and $\phi$ represent an external force and a heat source, respectively.

Received November 3, 2007.

2000 Mathematics Subject Classification. Primary 35P15; Secondary 34E10, 74F05.

(C) 2008 by the authors Reverts to public domain 28 years from publication 
For a finite string, it is not restrictive to assume that the reference configuration is the interval $(a, b)$ with $a<0<b$. We are interested in local perturbations of the mass density,

$$
\rho_{\varepsilon}(x)= \begin{cases}p(x) & \text { if } x \in(a,-\varepsilon) \cup(\varepsilon, b), \\ \varepsilon^{-m} q\left(\frac{x}{\varepsilon}\right) & \text { if } x \in(-\varepsilon, \varepsilon),\end{cases}
$$

represented by two parameters $\varepsilon \rightarrow 0$ and $m \in \mathbb{R}$. The mass density functions $p:[a, b] \rightarrow$ $\mathbb{R}$ and $q:[-1,1] \rightarrow \mathbb{R}$ are bounded and strictly positive. We assume that $p$ is continuous on $[a, 0)$ and $(0, b]$, while $q$ is continuous on $[-1,1]$. We also suppose that all other parameters are strictly positive in $\bar{\Omega}$ and smooth enough, namely $\alpha, \beta, \varkappa \in C^{1}(a, b)$, and $k \in C^{0}(a, b)$. In this note, we analyse the asymptotic behaviour as $\varepsilon \rightarrow 0$ of the eigenvalues $\lambda_{\varepsilon}$ and eigenvectors $\left(u_{\varepsilon}, \theta_{\varepsilon}\right)$ of the eigenvalue problem associated with (1D) and (2) on $\Omega_{\varepsilon}:=(a,-\varepsilon) \cup(-\varepsilon, \varepsilon) \cup(\varepsilon, b)$,

$$
\begin{aligned}
&-\left(\alpha(x) u_{\varepsilon}^{\prime}\right)^{\prime}+\left(\beta(x) \theta_{\varepsilon}\right)^{\prime}=-\lambda_{\varepsilon}^{2} \rho_{\varepsilon}(x) u_{\varepsilon}, \\
&-\left(\varkappa(x) \theta_{\varepsilon}^{\prime}\right)^{\prime}-\lambda_{\varepsilon} \beta(x) u_{\varepsilon}^{\prime}=\lambda_{\varepsilon} k(x) \theta_{\varepsilon},
\end{aligned}
$$

complemented with the Dirichlet boundary conditions at the outer ends

$$
u_{\varepsilon}(a)=u_{\varepsilon}(b)=0 \quad \text { and } \quad \theta_{\varepsilon}(a)=\theta_{\varepsilon}(b)=0,
$$

and the interfacial conditions

$$
\llbracket u_{\varepsilon} \rrbracket_{ \pm \varepsilon}=\llbracket u_{\varepsilon}^{\prime} \rrbracket_{ \pm \varepsilon}=0 \quad \text { and } \quad \llbracket \theta_{\varepsilon} \rrbracket_{ \pm \varepsilon}=\llbracket \theta_{\varepsilon}^{\prime} \rrbracket_{ \pm \varepsilon}=0 .
$$

The prime ' denotes the derivative with respect to the spatial variable. We use the notation $\llbracket y \rrbracket_{z}:=y(z+0)-y(z-0)$ for the jump of a function $y$ at point $z$. The smoothness assumptions imply that $u_{\varepsilon}$ and $\theta_{\varepsilon}$, given as solutions of the ordinary differential equations (3) and (44), belong to $C^{1}(a, b)$, and that their restrictions to the intervals $(a,-\varepsilon)$, $(-\varepsilon, \varepsilon)$ and $(\varepsilon, b)$ are twice continuously differentiable.

For $\beta=0$, (3)-(6) splits into two independent problems, with the perturbation being present only in the elastic part. The corresponding study of the asymptotic behaviour for eigenvalues and eigenfunctions in the isothermal elastic problem is presented in [4. We refer to 9, 10, for the first systematic study of problems with concentrated masses (see also [7, 3]). We remark that a thermoelastic setting has been investigated before [9, 8]; there, perturbations of thermal characteristics are investigated, while the present analysis focuses on the local perturbation of elastic coefficients and their influence on the thermal behaviour of the system.

Methodologically, the thermoelastic problem is different from the isothermal one. One of the differences is the non-selfadjointness of the corresponding operator, which renders the justification methods used for the isothermal problem [4 inapplicable. Even the existence of eigenvalues and eigenfunctions requires special analysis [12. Another main difference is that the operator of classical thermo-elasticity acts in a space of low regularity, which would not allow the application of methods developed for localised perturbations in [4. In this article, we construct the operator framework for thermoelastic problems in a space of higher regularity, namely $\left[H_{0}^{1}\right]^{3}$. The equality of the spectra and a correspondence of the eigenfunctions is established, which is a self-contained problem in its 
own right. Then the limit behaviour for the spectral properties and the rates of convergence are estimated for two classes of relatively light mass density perturbations, namely $m<1$ and $m=1$. The case $m=1$ amounts to the presence of a concentrated (finite and non-zero) mass in the limit, whereas $m<1$ corresponds to an unperturbed string in the limit. Within the scope of this article, we analyse these two cases of relatively light perturbations, rather than heavy mass perturbations $m>1$.

2. Operator frameworks. We note that (3)-(6) is an eigenvalue problem for a quadratic operator pencil. Introducing the independent variables $u_{1}^{\varepsilon}=u_{\varepsilon}, u_{2}^{\varepsilon}=-\lambda_{\varepsilon} u_{\varepsilon}$, $u_{3}^{\varepsilon}=\theta_{\varepsilon}$ we linearise problem (3) $-(\underline{6})$ :

$$
\begin{aligned}
-u_{2}^{\varepsilon} & =\lambda_{\varepsilon} u_{1}^{\varepsilon}, \\
-\left(\alpha\left(u_{1}^{\varepsilon}\right)^{\prime}\right)^{\prime}+\left(\beta u_{3}^{\varepsilon}\right)^{\prime} & =\lambda_{\varepsilon} \rho_{\varepsilon} u_{2}^{\varepsilon}, \\
-\left(\varkappa\left(u_{3}^{\varepsilon}\right)^{\prime}\right)^{\prime}+\beta\left(u_{2}^{\varepsilon}\right)^{\prime} & =\lambda_{\varepsilon} k u_{3}^{\varepsilon} \quad \text { for } \quad x \in \Omega_{\varepsilon}, \\
u_{j}^{\varepsilon}(a)=u_{j}^{\varepsilon}(b)=0 \text { and } \quad\left[\left[u_{j}^{\varepsilon}\right]\right]_{ \pm \varepsilon} & =\left[\left[\left(u_{j}^{\varepsilon}\right)^{\prime}\right]\right]_{ \pm \varepsilon}=0 \quad \text { for } j=1,2,3 .
\end{aligned}
$$

We use a standard notation for Lebesgue and Sobolev spaces: $L_{p}^{2}(\Omega)$ is a $p$-weighted $L^{2}$-space of square integrable functions in $\Omega, H^{n}(\Omega)$ is the space of functions whose distributional derivatives up to the order $n$ inclusive are in $L^{2}(\Omega)$, while $H_{0}^{1}(\Omega)$ consists of the functions in $H^{1}(\Omega)$ with a zero trace on the boundary $\partial \Omega$; the inner product in $H_{0}^{1}(\Omega)$ is $(u, v)_{H_{0}^{1}(\Omega)}:=\left(\alpha u^{\prime}, v^{\prime}\right)_{L^{2}(\Omega)}$. We introduce the Hilbert spaces

$$
\mathcal{L}_{\varepsilon}:=H_{0}^{1}(a, b) \times L_{\rho_{\varepsilon}}^{2}(a, b) \times L_{k}^{2}(a, b) \quad \text { and } \quad \mathcal{H}:=H_{0}^{1}(a, b) \times H_{0}^{1}(a, b) \times H_{0}^{1}(a, b),
$$

equipped with inner products for $U=\left(u_{1}, u_{2}, u_{3}\right)^{T}$ and $V=\left(v_{1}, v_{2}, v_{3}\right)^{T}$ (the bar denotes complex conjugation),

$$
(U, V)_{\mathcal{L}_{\varepsilon}}:=\int_{a}^{b}\left[\alpha(x) u_{1}^{\prime} \bar{v}_{1}^{\prime}+\rho_{\varepsilon}(x) u_{2} \bar{v}_{2}+k(x) u_{3} \bar{v}_{3}\right] \mathrm{d} x
$$

and

$$
(U, V)_{\mathcal{H}}:=\int_{a}^{b} \alpha(x)\left[u_{1}^{\prime} \bar{v}_{1}^{\prime}+u_{2}^{\prime} \bar{v}_{2}^{\prime}+u_{3}^{\prime} \bar{v}_{3}^{\prime}\right] \mathrm{d} x .
$$

We introduce an operator $\mathcal{A}_{\varepsilon}: \mathcal{L}_{\varepsilon} \rightarrow \mathcal{L}_{\varepsilon}$ with the domain $\mathfrak{D}\left(\mathcal{A}_{\varepsilon}\right)=\mathfrak{D}_{*} \times H_{0}^{1}(a, b) \times \mathfrak{D}_{*}$, where

$$
\mathfrak{D}_{*}:=H^{2}(a, b) \cap H_{0}^{1}(a, b)=\left\{y \in H^{2}(a, b) \mid y(a)=y(b)=0\right\},
$$

and with an action given by $\mathcal{A}_{\varepsilon} V:=A_{\varepsilon}\left(x, \frac{d}{d x}\right) V(x)$, where

$$
A_{\varepsilon}(x, \mathcal{D})=\left(\begin{array}{ccc}
0 & -I & 0 \\
-\rho_{\varepsilon}^{-1} \mathcal{D} \alpha \mathcal{D} & 0 & \rho_{\varepsilon}^{-1} \mathcal{D} \beta \\
0 & k^{-1} \beta \mathcal{D} & -k^{-1} \mathcal{D} \varkappa \mathcal{D}
\end{array}\right) .
$$

Then (7)-(10) become

$$
\mathcal{A}_{\varepsilon} U_{\varepsilon}=\lambda_{\varepsilon} U_{\varepsilon}
$$


The operator $\mathcal{A}_{\varepsilon}$ is classical for the problems in linearised thermoelasticity [9, 8]. Nevertheless, since $U_{\varepsilon} \in \mathcal{H}$ and $\mathcal{H}$ is more regular than $\mathcal{L}_{\varepsilon}$, for our purposes it is more convenient to consider (7)-(10) in $\mathcal{H}$. The higher regularity is necessary for the justification of the convergence and the estimation of error bounds as $\varepsilon \rightarrow 0$. Thus, we further construct an operator framework for (7)-(10) in $\mathcal{H}$.

A variational formulation of (12) is

$$
\left(\mathcal{A}_{\varepsilon} U_{\varepsilon}, \Phi\right)_{\mathcal{L}_{\varepsilon}}=\lambda_{\varepsilon}\left(U_{\varepsilon}, \Phi\right)_{\mathcal{L}_{\varepsilon}} \quad \text { for every } \Phi \in \mathcal{L}_{\varepsilon}
$$

which in the component-wise representation for $U_{\varepsilon}=\left(u_{1}^{\varepsilon}, u_{2}^{\varepsilon}, u_{3}^{\varepsilon}\right)$ and $\Phi=\left(\varphi_{1}, \varphi_{2}, \varphi_{3}\right)$ reads

$$
\begin{aligned}
-\int_{a}^{b} \alpha(x)\left(u_{2}^{\varepsilon}\right)^{\prime} \bar{\varphi}_{1}^{\prime} \mathrm{d} x & =\lambda_{\varepsilon} \int_{a}^{b} \alpha(x)\left(u_{1}^{\varepsilon}\right)^{\prime} \bar{\varphi}_{1}^{\prime} \mathrm{d} x, \\
\int_{a}^{b}\left[\alpha(x)\left(u_{1}^{\varepsilon}\right)^{\prime} \bar{\varphi}_{2}^{\prime}+\left(\beta(x) u_{3}^{\varepsilon}\right)^{\prime} \bar{\varphi}_{2}\right] \mathrm{d} x & =\lambda_{\varepsilon} \int_{a}^{b} \rho_{\varepsilon}(x) u_{2}^{\varepsilon} \bar{\varphi}_{2} \mathrm{~d} x, \\
\int_{a}^{b}\left[\varkappa(x)\left(u_{3}^{\varepsilon}\right)^{\prime} \bar{\varphi}_{3}^{\prime}+\beta(x)\left(u_{2}^{\varepsilon}\right)^{\prime} \bar{\varphi}_{3}\right] \mathrm{d} x & =\lambda_{\varepsilon} \int_{a}^{b} k(x) u_{3}^{\varepsilon} \bar{\varphi}_{3} \mathrm{~d} x .
\end{aligned}
$$

Note that the left-hand side of (14)-(16) is independent of $\varepsilon$. We introduce a sesquilinear form $\tau$ on $\mathcal{H}$,

$$
\tau(U, \Phi):=\int_{a}^{b}\left[-\alpha u_{2}^{\prime} \bar{\varphi}_{1}^{\prime}+\alpha u_{1}^{\prime} \bar{\varphi}_{2}^{\prime}+\left(\beta u_{3}\right)^{\prime} \bar{\varphi}_{2}+\beta u_{2}^{\prime} \bar{\varphi}_{3}+\varkappa u_{3}^{\prime} \bar{\varphi}_{3}^{\prime}\right] \mathrm{d} x,
$$

which represents the sum of the left-hand sides in (14)-(16). Since $\tau(\cdot, \cdot)$ is obviously continuous on $\mathcal{H}$, there exists by the Lax-Milgram theorem [11] a bounded operator $\mathcal{B}: \mathcal{H} \rightarrow \mathcal{H}$ such that $(\mathcal{B} U, \Phi)_{\mathcal{H}}=\tau(U, \Phi)$ for every $\Phi \in \mathcal{H}$. Thus the eigenvalue problem (14)-(16) can be written in the form

$$
\left(\mathcal{B} U_{\varepsilon}, \Phi\right)_{\mathcal{H}}=\lambda_{\varepsilon}\left(U_{\varepsilon}, \Phi\right)_{\mathcal{L}_{\varepsilon}} \text { for every } \Phi \in \mathcal{H}
$$

Likewise, since the sesquilinear form $(\cdot, \cdot)_{\mathcal{L}_{\varepsilon}}$ is continuous on $\mathcal{H}$, there exists, again by the Lax-Milgram theorem, a bounded operator $\mathcal{Q}_{\varepsilon}: \mathcal{H} \rightarrow \mathcal{H}$ such that

$$
\left(\mathcal{Q}_{\varepsilon} U, \Phi\right)_{\mathcal{H}}=(U, \Phi)_{\mathcal{L}_{\varepsilon}} \text { for every } \Phi \in \mathcal{H}
$$

Hence, (18) yields that the eigenvalue problem can be represented as

$$
\mathcal{B} U_{\varepsilon}=\lambda_{\varepsilon} \mathcal{Q}_{\varepsilon} U_{\varepsilon} \text { in } \mathcal{H} .
$$

The following sections analyse the equivalence of (20) and (12).

\section{Existence of inverse operators.}

Lemma 1. The inverse $\mathcal{B}^{-1}$ exists and is a bounded operator in $\mathcal{H}$.

Proof. We need to prove that for every $F \in \mathcal{H}$, there exists a unique solution $U \in \mathcal{H}$ to the problem $\mathcal{B} U=F$. The weak formulation of this problem is to find, for arbitrary $F \in \mathcal{H}, U \in \mathcal{H}$ such that

$$
\tau(U, \Phi)=(F, \Phi)_{\mathcal{H}} \text { for every } \Phi \in \mathcal{H} .
$$


A consecutive substitution of the test functions $\Phi=\left(\phi_{1}, 0,0\right)^{T}, \Phi=\left(0, \phi_{2}, 0\right)^{T}$ and $\Phi=\left(0,0, \phi_{3}\right)^{T}$ into (21) yields

$$
\begin{array}{rr}
-\int_{a}^{b} \alpha(x) u_{2}^{\prime} \bar{\varphi}_{1}^{\prime} \mathrm{d} x=\int_{a}^{b} \alpha(x) f_{1}^{\prime} \bar{\varphi}_{1}^{\prime} \mathrm{d} x & \text { for every } \varphi_{1} \in H_{0}^{1}(a, b), \\
\int_{a}^{b}\left[\alpha(x) u_{1}^{\prime} \bar{\varphi}_{2}^{\prime}+\left(\beta(x) u_{3}\right)^{\prime} \bar{\varphi}_{2}\right] \mathrm{d} x=\int_{a}^{b} \alpha(x) f_{2}^{\prime} \bar{\varphi}_{2}^{\prime} \mathrm{d} x & \text { for every } \varphi_{2} \in H_{0}^{1}(a, b), \\
\int_{a}^{b}\left[\varkappa(x) u_{3}^{\prime} \bar{\varphi}_{3}^{\prime}+\beta(x) u_{2}^{\prime} \bar{\varphi}_{3}\right] \mathrm{d} x=\int_{a}^{b} \alpha(x) f_{3}^{\prime} \bar{\varphi}_{3}^{\prime} \mathrm{d} x & \text { for every } \varphi_{3} \in H_{0}^{1}(a, b) .
\end{array}
$$

Then (22) implies $u_{2}=-f_{1} \in H_{0}^{1}(a, b)$. Obviously,

$$
\left\|u_{2}\right\|_{H_{0}^{1}(a, b)}=\left\|f_{1}\right\|_{H_{0}^{1}(a, b)} \leq\|F\|_{\mathcal{H}} .
$$

Hence, (24) becomes

$$
\int_{a}^{b} \varkappa(x) u_{3}^{\prime} \bar{\varphi}_{3}^{\prime} \mathrm{d} x=\int_{a}^{b}\left[\beta(x) f_{1}^{\prime} \bar{\varphi}_{3}+\alpha(x) f_{3}^{\prime} \bar{\varphi}_{3}^{\prime}\right] \mathrm{d} x \quad \text { for every } \phi_{3} \in H_{0}^{1}(a, b) .
$$

Since for each fixed pair $\left(f_{1}, f_{3}\right)$ the right-hand side of (26) is a semilinear bounded functional on $H_{0}^{1}(a, b)$, the Lax-Milgram theorem provides the existence of a unique $u_{3} \in H_{0}^{1}(a, b)$ for any such pair. Moreover, by substituting $\varphi_{3}=u_{3}$ we immediately find

$$
\left\|u_{3}\right\|_{H_{0}^{1}(a, b)}^{2} \leq C\left|\left(\beta f_{1}^{\prime}, u_{3}\right)_{L^{2}(a, b)}\right|+\left|\left(\alpha f_{3}^{\prime}, u_{3}^{\prime}\right)_{L^{2}(a, b)}\right| \leq C\|F\|_{\mathcal{H}}\left\|u_{3}\right\|_{H_{0}^{1}(a, b)},
$$

with a positive constant $C$ independent of $F$. We follow the convention of writing $C$ for a generic constant whose value may change from line to line. Estimate (27) implies

$$
\left\|u_{3}\right\|_{H_{0}^{1}(a, b)} \leq C\|F\|_{\mathcal{H}} .
$$

Proceeding in a similar fashion for $u_{1}$ in (23), we find after a straightforward calculation that

$$
\left\|u_{1}\right\|_{H_{0}^{1}(a, b)} \leq C\|F\|_{\mathcal{H}} .
$$

Thus, for arbitrary $F=\left(f_{1}, f_{2}, f_{3}\right)^{T}$, there is a unique $U=\left(u_{1}, u_{2}, u_{3}\right)^{T}$ such that $\mathcal{B} U=F$. Moreover, the estimates (25), (28) and (29) imply that $\|U\|_{\mathcal{H}} \leq C\|F\|_{\mathcal{H}}$. Therefore, the operator $\mathcal{B}^{-1}$ exists and is bounded.

We note that, as a result of Lemma 1, the eigenvalue problem (20) can be formulated equivalently as

$$
U_{\varepsilon}=\lambda_{\varepsilon} \mathcal{M}_{\varepsilon} U_{\varepsilon} \text { in } \mathcal{H}
$$

with the operator $\mathcal{M}_{\varepsilon}:=\mathcal{B}^{-1} \mathcal{Q}_{\varepsilon}$. It follows that $\lambda_{\varepsilon} \neq 0$, since $\lambda_{\varepsilon}=0$ immediately implies $U_{\varepsilon}=0$ in contradiction to the definition of an eigenvector. Thus, $\lambda_{\varepsilon}^{-1}$ exists, and (30) allows the representation $\mathcal{M}_{\varepsilon} U_{\varepsilon}=\lambda_{\varepsilon}^{-1} U_{\varepsilon}$ in $\mathcal{H}$.

Lemma 2. The inverse operator $\mathcal{A}_{\varepsilon}^{-1}: \mathcal{L}_{\varepsilon} \rightarrow \mathcal{L}_{\varepsilon}$ exists and is compact. 
Proof. The existence of $\mathcal{A}_{\varepsilon}^{-1}$ is equivalent to the existence of a solution $U_{\varepsilon}$ to the problem $\mathcal{A}_{\varepsilon} U_{\varepsilon}=F$ for any $F \in \mathcal{L}_{\varepsilon}$, which reads component-wise as

$$
\begin{aligned}
-u_{2}^{\varepsilon} & =f_{1} \quad \text { in } \Omega_{\varepsilon}, & u_{2}^{\varepsilon} \in H_{0}^{1}(a, b), \\
-\left(\alpha\left(u_{1}^{\varepsilon}\right)^{\prime}\right)^{\prime}+\left(\beta u_{3}^{\varepsilon}\right)^{\prime}=\rho_{\varepsilon} f_{2} & \text { in } \Omega_{\varepsilon}, & u_{1}^{\varepsilon} \in \mathfrak{D}_{*}, \\
-\left(\varkappa\left(u_{3}^{\varepsilon}\right)^{\prime}\right)^{\prime}+\beta\left(u_{2}^{\varepsilon}\right)^{\prime}=k f_{3} & \text { in } \Omega_{\varepsilon}, & u_{3}^{\varepsilon} \in \mathfrak{D}_{*} .
\end{aligned}
$$

The argument is similar to that proposed for the proof of Lemma 1. We first find $u_{2}^{\varepsilon}=-f_{1} \in H_{0}^{1}(a, b)$ from (31) such that

$$
\left\|u_{2}^{\varepsilon}\right\|_{H_{0}^{1}(a, b)}=\left\|f_{1}\right\|_{H_{0}^{1}(a, b)} \leq\|F\|_{\mathcal{L}_{\varepsilon}} .
$$

Next, from (33) we find $u_{3}^{\varepsilon} \in H^{2}(a, b) \cap H_{0}^{1}(a, b)$ such that $\left(\varkappa\left(u_{3}^{\varepsilon}\right)^{\prime}\right)^{\prime}=\beta\left(u_{2}^{\varepsilon}\right)^{\prime}-k f_{3} \in$ $L^{2}(a, b)$, which yields

$$
\left\|u_{3}^{\varepsilon}\right\|_{H_{0}^{1}(a, b)}=C\left(\left\|u_{2}^{\varepsilon}\right\|_{H_{0}^{1}(a, b)}+\left\|f_{3}\right\|_{L_{k}^{2}(a, b)}\right) \leq C\|F\|_{\mathcal{L}_{\varepsilon}} .
$$

Finally, we find from (32), $u_{1}^{\varepsilon} \in H^{2}(a, b) \cap H_{0}^{1}(a, b)$ such that $\left(\alpha\left(u_{1}^{\varepsilon}\right)^{\prime}\right)^{\prime}=\left(\beta u_{3}^{\varepsilon}\right)^{\prime}-\rho_{\varepsilon} f_{2} \in$ $L^{2}(a, b)$, which yields $\left\|u_{1}^{\varepsilon}\right\|_{H_{0}^{1}(a, b)} \leq C\|F\|_{\mathcal{L}_{\varepsilon}}$ and, moreover,

$$
\left\|u_{1}^{\varepsilon}\right\|_{H^{2}(a, b)}=C\left(\left\|u_{1}^{\varepsilon}\right\|_{H_{0}^{1}(a, b)}+\left\|u_{3}^{\varepsilon}\right\|_{H_{0}^{1}(a, b)}+\left\|f_{2}\right\|_{L_{\rho_{\varepsilon}}^{2}(a, b)}\right) \leq C\|F\|_{\mathcal{L}_{\varepsilon}} .
$$

Thus, the solution $U_{\varepsilon}=\left(u_{1}^{\varepsilon}, u_{2}^{\varepsilon}, u_{3}^{\varepsilon}\right)^{T}$ of (31)-(33) can be represented as a bounded operator $\hat{A}_{\varepsilon}^{-1}: \mathcal{L}_{\varepsilon} \rightarrow \mathcal{H}_{2}$, where $\mathcal{H}_{2}=H^{2}(a, b) \times H_{0}^{1}(a, b) \times H_{0}^{1}(a, b)$. Then the operator $\mathcal{A}_{\varepsilon}^{-1}: \mathcal{L}_{\varepsilon} \rightarrow \mathcal{L}_{\varepsilon}$ exists and can be represented as $\mathcal{A}_{\varepsilon}^{-1}=J_{*} \hat{A}_{\varepsilon}^{-1}$, where $J_{*}: \mathcal{H}_{2} \rightarrow \mathcal{L}_{\varepsilon}$ is a compact embedding operator

$$
J_{*}=\left(\begin{array}{ccc}
J & 0 & 0 \\
0 & J_{1} & 0 \\
0 & 0 & J_{1}
\end{array}\right)
$$

with compact embedding components $J: H^{2}(a, b) \rightarrow H^{1}(a, b), J_{1}: H_{0}^{1}(a, b) \rightarrow L^{2}(a, b)$. Therefore, $\mathcal{A}_{\varepsilon}^{-1}$ is compact as a product of a bounded and a compact operator.

The compactness of $\mathcal{A}_{\varepsilon}^{-1}$ yields the following result (see [6, Theorem III.6.29]).

Corollary 1. The spectrum $\sigma\left(\mathcal{A}_{\varepsilon}\right)$ of $\mathcal{A}_{\varepsilon}$ is no more than a countable set of isolated eigenvalues with finite multiplicity. There is no accumulation point of the spectrum other than infinity. The eigenprojector $\mathcal{P}_{\varepsilon}$ to the generalised eigenspace corresponding to each $\lambda_{\varepsilon} \in \sigma\left(\mathcal{A}_{\varepsilon}\right)$ is identical with the eigenprojector corresponding to the eigenvalue $\lambda_{\varepsilon}^{-1}$ of $\mathcal{A}_{\varepsilon}^{-1}$. Moreover, if $\lambda_{\varepsilon} \in \sigma\left(\mathcal{A}_{\varepsilon}\right)$, then $\bar{\lambda}_{\varepsilon} \in \sigma\left(\mathcal{A}_{\varepsilon}^{*}\right)$ has the same multiplicity, both in the geometric and the algebraic sense by the Riesz-Schauder Theorem [6, Remark 6.27] (the star $^{*}$ denotes the adjoint operator).

4. Equivalence of eigenvalue problems. The aim of this section is to establish the equivalence between the spectral properties of the operators $\mathcal{M}_{\varepsilon}:=\mathcal{B}^{-1} \mathcal{Q}_{\varepsilon}$ and $\mathcal{A}_{\varepsilon}$ in the sense of Lemma 6. Since the operators are defined on two different Hilbert spaces, we first formulate auxiliary statements in Lemmas 35

Let the operators $\mathcal{P}_{j}: \mathcal{H} \rightarrow H_{0}^{1}(a, b)$ be given by $\mathcal{P}_{j} U=u_{j}$ for $U=\left(u_{1}, u_{2}, u_{3}\right)^{T}$, $j=1,2,3$. 
Lemma 3. For every $V \in \mathcal{H}$, the images $\mathcal{P}_{1} \mathcal{M}_{\varepsilon} V$ and $\mathcal{P}_{3} \mathcal{M}_{\varepsilon} V$ are functions in $H^{2}(a, b)$.

Proof. First, we show that the operator $\mathcal{Q}_{\varepsilon}$ defined by (19) admits the representation

$$
\mathcal{Q}_{\varepsilon}=\left(\begin{array}{ccc}
I_{1} & 0 & 0 \\
0 & J Q_{1}^{\varepsilon} & 0 \\
0 & 0 & J Q_{2}
\end{array}\right),
$$

where $I_{1}: H_{0}^{1}(a, b) \rightarrow H_{0}^{1}(a, b)$ is the identity operator, $J: H^{2}(a, b) \rightarrow H^{1}(a, b)$ is a compact embedding operator and the operators $Q_{1}^{\varepsilon}$ and $Q_{2}$ are as follows. The operator $Q_{1}^{\varepsilon}: H_{0}^{1}(a, b) \rightarrow H^{2}(a, b)$ gives a solution $w_{1}=Q_{1}^{\varepsilon} u$ to the problem

$$
-\left(\alpha w_{1}^{\prime}\right)^{\prime}=\rho_{\varepsilon} u \quad \text { in }(a, b), \quad \llbracket w_{1} \rrbracket_{ \pm \varepsilon}=\llbracket w_{1}^{\prime} \rrbracket_{ \pm \varepsilon}=0, \quad w_{1}(a)=w_{1}(b)=0 .
$$

The operator $Q_{2}: H_{0}^{1}(a, b) \rightarrow H^{2}(a, b)$ gives a solution $w_{2}=Q_{2} u$ to the problem

$$
-\left(\alpha w_{2}^{\prime}\right)^{\prime}=k u \text { in }(a, b), \quad w_{2}(a)=w_{2}(b)=0 .
$$

Since the weak formulations of (37) and (38) are such that for any $\varphi_{1}, \varphi_{2} \in H_{0}^{1}(a, b)$,

$$
\int_{a}^{b} \alpha w_{1}^{\prime} \varphi_{1}^{\prime} \mathrm{d} x=\int_{a}^{b} \rho_{\varepsilon} w_{1} \varphi_{1} \mathrm{~d} x \text { and } \int_{a}^{b} \alpha w_{2}^{\prime} \varphi_{2}^{\prime} \mathrm{d} x=\int_{a}^{b} k w_{2} \varphi_{2} \mathrm{~d} x
$$

the operator given by the representation (36) coincides with (19). Clearly, $Q_{1}^{\varepsilon}$ and $Q_{2}$ are bounded, $\left\|Q_{1}^{\varepsilon}\right\| \leq C_{\varepsilon},\left\|Q_{2}\right\| \leq C$, with positive constants $C_{\varepsilon}$ and $C$. Therefore $J Q_{1}^{\varepsilon}$ and $J Q_{2}$ are compact for any fixed $\varepsilon>0$.

Second, we show that in any vector of the form $U_{\varepsilon}=\mathcal{M}_{\varepsilon} V=\mathcal{B}^{-1} \mathcal{Q}_{\varepsilon} V$ the first and the third components belong to $H^{2}(a, b)$. Note that $U_{\varepsilon} \in \mathcal{H}$ solves (22)-(24) with $F=\mathcal{Q}_{\varepsilon} V=\left(v_{1}, J Q_{1}^{\varepsilon} v_{2}, J Q_{2} v_{3}\right)^{T}$. Then (22) yields

$$
u_{2}=-v_{1} \in H_{0}^{1}(a, b) \text {. }
$$

Since $Q_{1}^{\varepsilon} v_{2}$ and $Q_{2} v_{3}$ belong to $H^{2}(a, b)$ and (24) implies

$$
\int_{a}^{b} \varkappa u_{3}^{\prime} \bar{\varphi}_{3}^{\prime} \mathrm{d} x=\int_{a}^{b}\left[-\left(\alpha\left(Q_{2} v_{3}\right)^{\prime}\right)^{\prime}+\beta v_{1}^{\prime}\right] \bar{\varphi}_{3} \mathrm{~d} x \quad \text { for every } \phi_{3} \in H_{0}^{1}(a, b),
$$

we obtain for the distributional derivatives

$$
\left(\varkappa u_{3}^{\prime}\right)^{\prime}=\left(\alpha\left(Q_{2} v_{3}\right)^{\prime}\right)^{\prime}+\beta u_{2}^{\prime} \in L^{2}(a, b) .
$$

Since $\varkappa \in C^{1}(a, b)$ is strictly positive, $u_{3}=\mathcal{P}_{3} U_{\varepsilon} \in H_{0}^{1}(a, b)$ along with (41) provides $u_{3} \in H^{2}(a, b)$. Then identity (23) yields $\int_{a}^{b} \alpha u_{1}^{\prime} \bar{\varphi}_{2}^{\prime} \mathrm{d} x=\int_{a}^{b}\left[-\left(\alpha\left(Q_{1}^{\varepsilon} v_{2}\right)^{\prime}\right)^{\prime}-\left(\beta u_{3}\right)^{\prime}\right] \bar{\varphi}_{2} \mathrm{~d} x$ for every $\phi_{2} \in H_{0}^{1}(a, b)$. Thus

$$
\left(\alpha u_{1}^{\prime}\right)^{\prime}=\left(\alpha\left(Q_{1}^{\varepsilon} v_{2}\right)^{\prime}\right)^{\prime}+\left(\beta u_{3}\right)^{\prime} \in L^{2}(a, b) .
$$

This in turn implies with $u_{1}=\mathcal{P}_{1} U_{\varepsilon} \in H_{0}^{1}(a, b)$ and the strict positivity of $\alpha \in C^{1}(a, b)$ that $u_{1} \in H^{2}(a, b)$.

Let $\mathfrak{R}\left(\mathcal{M}_{\varepsilon}\right)$ be the range of $\mathcal{M}_{\varepsilon}$, and let $J_{* *}: \mathcal{H} \rightarrow \mathcal{L}_{\varepsilon}$ be an embedding operator

$$
J_{* *}=\left(\begin{array}{ccc}
I & 0 & 0 \\
0 & J_{1} & 0 \\
0 & 0 & J_{1}
\end{array}\right)
$$

with the identity operator $I$ and $J_{1}$ as above. 
Lemma 4. The following embedding of the functional sets:

$$
\mathfrak{R}\left(\mathcal{M}_{\varepsilon}\right) \subset \mathfrak{D}\left(\mathcal{A}_{\varepsilon}\right)=\mathfrak{R}\left(\mathcal{A}_{\varepsilon}^{-1}\right) \subset \mathcal{H}=\mathfrak{D}\left(\mathcal{M}_{\varepsilon}\right) \subset \mathcal{L}_{\varepsilon}=\mathfrak{D}\left(\mathcal{A}_{\varepsilon}^{-1}\right)
$$

holds, along with the operator equality $\mathcal{A}_{\varepsilon}^{-1} J_{* *}=J_{* *} \mathcal{M}_{\varepsilon}$.

Proof. The embedding (43) is a corollary to Lemma 3 and the definitions of the involved operators.

Let us prove the operator equality. Note that the domains of the operators are equal, $\mathfrak{D}\left(\mathcal{A}_{\varepsilon}^{-1} J_{* *}\right)=\mathcal{H}=\mathfrak{D}\left(J_{* *} \mathcal{M}_{\varepsilon}\right)$. We now prove that for every $V \in \mathcal{H}$, the functions $U_{\varepsilon}^{\text {left }}:=\mathcal{A}_{\varepsilon}^{-1} J_{* *} V$ and $U_{\varepsilon}^{\text {right }}:=\mathcal{M}_{\varepsilon} V$ coincide up to the embedding operator $J_{* *}$. We set $F:=J_{* *} V$. Then $U_{\varepsilon}^{\text {left }}$ is a unique solution to problem (31)-(33). On the other hand, $U_{\varepsilon}^{\text {right }}$ is a unique solution to (39), (41), (42) along with Dirichlet boundary conditions. The idea is to show that the problems coincide for the given data. Note that (31) and (39) are equivalent. The equivalence of (33) and (41) with Dirichlet conditions follows from the definition of the operator $Q_{2}$ in (38). Similarly, the equivalence of (32) and (42) is guaranteed by the definition of $Q_{1}^{\varepsilon}$ in (37). By the uniqueness of the solution, $U_{\varepsilon}^{\text {left }}$ and $U_{\varepsilon}^{\text {right }}$ coincide. Of course, they are equal as elements of $\mathcal{L}_{\varepsilon}$ after an application of the embedding operator $J_{* *}$.

Lemma 5. $\mathfrak{R}\left(\mathcal{M}_{\varepsilon}\right)$ is dense in $\mathcal{H}$.

Proof. We argue by contradiction. Let us suppose there is a non-zero $Y \in \mathcal{H}$ such that $(Y, \Phi)_{\mathcal{H}}=0$ for all $\Phi \in \mathfrak{R}\left(\mathcal{M}_{\varepsilon}\right)$. For an arbitrary $U \in \mathcal{H}$, consider

$$
0=\left(\mathcal{M}_{\varepsilon} U, Y\right)_{\mathcal{H}}=\left(\mathcal{B}^{-1} \mathcal{Q}_{\varepsilon} U, Y\right)_{\mathcal{H}}=\left(\mathcal{Q}_{\varepsilon} U,\left(\mathcal{B}^{-1}\right)^{*} Y\right)_{\mathcal{H}}=\left(U,\left(\mathcal{B}^{-1}\right)^{*} Y\right)_{\mathcal{L}_{\varepsilon}} .
$$

Since the latter is zero for any $U \in \mathcal{H}$, which is dense in $\mathcal{L}_{\varepsilon}$, we obtain $\left(\mathcal{B}^{-1}\right)^{*} Y=0$. Since both $\mathcal{B}$ and $\mathcal{B}^{-1}$ are bounded operators on $\mathcal{H}$, the kernel of $\left(\mathcal{B}^{-1}\right)^{*}$ is trivial. Therefore, $\left(\mathcal{B}^{-1}\right)^{*} Y=0$ implies $Y=0$ in contradiction to our assumption.

Lemma 5 allows us to introduce an operator $\mathcal{G}_{\varepsilon}=\mathcal{M}_{\varepsilon}^{-1}: \mathcal{H} \rightarrow \mathcal{H}$ with dense domain $\mathfrak{D}\left(\mathcal{G}_{\varepsilon}\right)=\mathfrak{R}\left(\mathcal{M}_{\varepsilon}\right)$. Note that according to (43), all eigenvectors $U_{\varepsilon}$ of $\mathcal{A}_{\varepsilon}$ belong to $\mathcal{L}_{\varepsilon} \cap \mathcal{H}$. In the next lemma, in order to emphasise the different functional classes, we use different notation for an eigenvector $U_{\varepsilon}$ as an element of $\mathcal{L}_{\varepsilon}$ and for its equivalent $V_{\varepsilon}$ in $\mathcal{H}$; the correspondence is then $U_{\varepsilon}=J_{* *} V_{\varepsilon}$. A pair consisting of an eigenvalue $\lambda_{\varepsilon}$ and a corresponding eigenfunction $U_{\varepsilon}$ of a given operator is referred to as an eigenmode of the operator.

Lemma 6. The following statements are equivalent, with $U_{\varepsilon}=J_{* *} V_{\varepsilon}$.

i) $\left(\lambda_{\varepsilon}, V_{\varepsilon}\right)$ is an eigenmode of $\mathcal{G}_{\varepsilon}=\mathcal{M}_{\varepsilon}^{-1}$;

ii) $\left(\lambda_{\varepsilon}^{-1}, V_{\varepsilon}\right)$ is an eigenmode of $\mathcal{M}_{\varepsilon}$;

iii) $\left(\lambda_{\varepsilon}^{-1}, U_{\varepsilon}\right)$ is an eigenmode of $\mathcal{A}_{\varepsilon}^{-1}$;

iv) $\left(\lambda_{\varepsilon}, U_{\varepsilon}\right)$ is an eigenmode of $\mathcal{A}_{\varepsilon}$.

Proof. The trivial equivalence $i i i) \Leftrightarrow i v$ ) is already stated in Corollary 1. The statement $i) \Leftrightarrow i i)$ is also immediate. We now prove $i i) \Rightarrow i i i)$. Let $\mu_{\varepsilon}=\lambda_{\varepsilon}^{-1}$. We apply $J_{* *}$ to both sides of $\mathcal{M}_{\varepsilon} V_{\varepsilon}=\mu_{\varepsilon} V_{\varepsilon}$. Then, by Lemma 4. $\mathcal{A}_{\varepsilon}^{-1} J_{* *} V_{\varepsilon}=\mu_{\varepsilon} J_{* *} V_{\varepsilon}$. Obviously if $V_{\varepsilon} \neq 0$, then $J_{* *} V_{\varepsilon} \neq 0$. We finally show $\left.\left.i i i\right) \Rightarrow i i\right)$. Note that if $U_{\varepsilon}$ is an eigenvector of 
$\mathcal{A}_{\varepsilon}^{-1}$

$$
\mathcal{A}_{\varepsilon}^{-1} U_{\varepsilon}=\mu_{\varepsilon} U_{\varepsilon}
$$

then $U_{\varepsilon} \in \mathfrak{R}\left(\mathcal{A}_{\varepsilon}^{-1}\right)$. Moreover, (43) provides $\mathfrak{R}\left(\mathcal{A}_{\varepsilon}^{-1}\right) \subset \mathcal{H}$. Then $U_{\varepsilon} \in \mathcal{L}_{\varepsilon} \cap \mathcal{H}$ can be presented in the form $U_{\varepsilon}=J_{* *} V_{\varepsilon}$ with $V_{\varepsilon} \in \mathcal{H}$. Again applying Lemma 4 to (44), we obtain $J_{* *} \mathcal{M}_{\varepsilon} V_{\varepsilon}=J_{* *} \mu_{\varepsilon} V_{\varepsilon}$. Since both $\mu_{\varepsilon} V_{\varepsilon}$ and $\mathcal{M}_{\varepsilon} V_{\varepsilon}$ belong to $\mathcal{H} \subset \mathcal{L}_{\varepsilon}$ and their traces in $\mathcal{L}_{\varepsilon}$ coincide, they are equal also as elements of $\mathcal{H}$, namely $\mathcal{M}_{\varepsilon} V_{\varepsilon}=\mu_{\varepsilon} V_{\varepsilon}$.

5. Spectral properties. The discreteness of the spectra of $\mathcal{A}_{\varepsilon}$ and $\mathcal{G}_{\varepsilon}$ is guaranteed by Corollary 1 and Lemma 6 . In this section, we discuss some additional properties of the spectra of the operator $\mathcal{A}_{\varepsilon}$ (and thus $\mathcal{G}_{\varepsilon}$ ) for fixed $\varepsilon>0$.

The completeness of generalised eigenfunctions for linear thermoelasticity has been proved by Yakubov [12] in the three-dimensional case. The proof can be easily adapted for one space dimension. In this case, a crucial ingredient, that is, a decay rate for approximation numbers, holds in an even stronger form [1].

Lemma 7. Each eigenvalue $\lambda_{\varepsilon}$ has a positive real part, $\operatorname{Re} \lambda_{\varepsilon}>0$.

Proof. We find from (18) that for a non-zero eigenvector $U_{\varepsilon}=\left(u_{1}^{\varepsilon}, u_{2}^{\varepsilon}, u_{3}^{\varepsilon}\right)$ corresponding to $\lambda_{\varepsilon}$,

$$
\lambda_{\varepsilon}=\frac{\left(\mathcal{B} U_{\varepsilon}, U_{\varepsilon}\right)_{\mathcal{H}}}{\left(U_{\varepsilon}, U_{\varepsilon}\right)_{\mathcal{L}_{\varepsilon}}}=\frac{\tau\left(U_{\varepsilon}, U_{\varepsilon}\right)}{\left(U_{\varepsilon}, U_{\varepsilon}\right)_{\mathcal{L}_{\varepsilon}}}=\frac{\tau\left(U_{\varepsilon}, U_{\varepsilon}\right)}{\left\|U_{\varepsilon}\right\|_{\mathcal{L}_{\varepsilon}}^{2}} .
$$

Since for any $U \in \mathcal{H}$, by the definition (17) of $\tau$,

we obtain

$$
\tau(U, U)=\int_{a}^{b}\left[\varkappa\left|u_{3}^{\prime}\right|^{2}+2 i \operatorname{Im}\left(\alpha u_{1}^{\prime} \bar{u}_{2}^{\prime}+\beta u_{2}^{\prime} \bar{u}_{3}\right)\right] \mathrm{d} x,
$$

$$
\operatorname{Re} \lambda_{\varepsilon}=\left\|U_{\varepsilon}\right\|_{\mathcal{L}_{\varepsilon}}^{-2} \int_{a}^{b} \varkappa\left|\left(u_{3}^{\varepsilon}\right)^{\prime}\right|^{2} \mathrm{~d} x
$$

Then obviously $\operatorname{Re} \lambda_{\varepsilon} \geq 0$. Let us show that $\operatorname{Re} \lambda_{\varepsilon} \neq 0$. Suppose to the contrary $\operatorname{Re} \lambda_{\varepsilon}=0$; we then find from (45) that $\left(u_{3}^{\varepsilon}\right)^{\prime}=0$ on $(a, b)$. Therefore, from (10) we find $u_{3}^{\varepsilon}=0$ on $(a, b)$. Since $\beta>0$, equation (9) yields $\left(u_{2}^{\varepsilon}\right)^{\prime}=0$ on $(a, b)$; again (10) gives $u_{2}^{\varepsilon}=0$ on $(a, b)$. Similarly, (8) along with (9) yields $u_{1}^{\varepsilon}=0$ on $(a, b)$, and thus $U_{\varepsilon}=0$ in contradiction to the assumption.

Lemma 8. If $U_{\varepsilon}$ is an eigenvector of $\mathcal{A}_{\varepsilon}$, then each component of the vector $U_{\varepsilon}$ is different from zero.

Proof. The proof of Lemma 7 shows that $u_{3}^{\varepsilon} \neq 0$. The remaining arguments are similar. If $u_{2}^{\varepsilon}=0$, then (7) would yield $u_{1}^{\varepsilon}=0$ and consequently (8) along with (10) implies $u_{3}^{\varepsilon}=0$, a contradiction. Thus $u_{2}^{\varepsilon} \neq 0$. If $u_{1}^{\varepsilon}=0$, then (7) immediately yields $u_{2}^{\varepsilon}=0$. Then (8) and (10) yield $u_{3}^{\varepsilon}=0$, again a contradiction.

Lemma 9. The adjoint operator admits the representation $\mathcal{A}_{\varepsilon}^{*}=\mathcal{T} \mathcal{A}_{\varepsilon} \mathcal{T}$ with

$$
\mathcal{T}=\left(\begin{array}{ccc}
1 & 0 & 0 \\
0 & -1 & 0 \\
0 & 0 & 1
\end{array}\right) \quad \text { and } \quad \mathfrak{D}\left(\mathcal{A}_{\varepsilon}^{*}\right)=\mathfrak{D}\left(\mathcal{A}_{\varepsilon}\right)
$$


Proof. Since the form

$$
(\mathcal{A} U, V)_{\mathcal{H}}=-\int_{a}^{b} \alpha u_{2}^{\prime} \bar{v}_{1}^{\prime} \mathrm{d} x+\int_{a}^{b}\left[-\left(\alpha u_{1}^{\prime}\right)^{\prime}+\left(\beta u_{3}\right)^{\prime}\right] \bar{v}_{2} \mathrm{~d} x+\int_{a}^{b}\left[-\left(\varkappa u_{3}^{\prime}\right)^{\prime}+\beta u_{2}^{\prime}\right] \bar{v}_{3} \mathrm{~d} x
$$

for all $U \in \mathfrak{D}(\mathcal{A})$ can be represented as

$$
(U, W)_{\mathcal{H}}=\int_{a}^{b} \alpha u_{1}^{\prime} \bar{w}_{1}^{\prime} \mathrm{d} x+\int_{a}^{b} \rho_{\varepsilon} u_{2} \bar{w}_{2} \mathrm{~d} x+\int_{a}^{b} k u_{3} \bar{w}_{3} \mathrm{~d} x
$$

only with $w_{1}=v_{2}, w_{2}=\frac{1}{\rho_{\varepsilon}}\left(\left(\alpha v_{1}^{\prime}\right)^{\prime}-\left(\beta v_{3}\right)^{\prime}\right), w_{3}=-\frac{1}{k}\left(\left(\varkappa v_{3}^{\prime}\right)^{\prime}+\beta v_{2}^{\prime}\right)$, for $v_{2}(a)=$ $v_{2}(b)=0$ and $v_{3}(a)=v_{3}(b)=0$, then by the definition of the adjoint operator, $W=\mathcal{A}_{\varepsilon}^{*} V$ with

$$
\mathcal{A}_{\varepsilon}^{*} V=A_{\varepsilon}^{*}\left(x, \frac{d}{d x}\right) V \quad \text { given by } A_{\varepsilon}^{*}(x, \mathcal{D})=\left(\begin{array}{ccc}
0 & I & 0 \\
\rho_{\varepsilon}^{-1} \mathcal{D} \alpha \mathcal{D} & 0 & -\rho_{\varepsilon}^{-1} \mathcal{D} \beta \\
0 & -k^{-1} \beta \mathcal{D} & -k^{-1} \mathcal{D} \varkappa \mathcal{D}
\end{array}\right) \text {. }
$$

Note again that the matrix-differential expression $A_{\varepsilon}^{*}\left(x, \frac{d}{d x}\right)$ can be applied only to the functions from $\mathfrak{D}_{*} \times H_{0}^{1}(a, b) \times \mathfrak{D}_{*}$. Thus $\mathfrak{D}\left(\mathcal{A}_{\varepsilon}^{*}\right)$ coincides with $\mathfrak{D}\left(\mathcal{A}_{\varepsilon}\right)$. The identity $\mathcal{A}_{\varepsilon}^{*}=\mathcal{T} \mathcal{A}_{\varepsilon} \mathcal{T}$ is easy to check.

REMARK 1 . One can see that the operator $\mathcal{A}_{\varepsilon}$ is not a normal operator for $\beta>0$, i.e., $\mathcal{A}_{\varepsilon}^{*} \mathcal{A}_{\varepsilon} \neq \mathcal{A}_{\varepsilon} \mathcal{A}_{\varepsilon}^{*}$. Since we are not using this fact, the proof is not presented here.

Lemma 10. If $\lambda_{\varepsilon} \in \sigma\left(\mathcal{A}_{\varepsilon}\right)$, then $\bar{\lambda}_{\varepsilon} \in \sigma\left(\mathcal{A}_{\varepsilon}\right)$ as well. Thus $\sigma\left(\mathcal{A}_{\varepsilon}\right)=\sigma\left(\mathcal{A}_{\varepsilon}^{*}\right)$.

Proof. If $\lambda_{\varepsilon} \in \sigma\left(\mathcal{A}_{\varepsilon}\right)$, then $\bar{\lambda}_{\varepsilon} \in \sigma\left(\mathcal{A}_{\varepsilon}^{*}\right)$ and thus $\mathcal{A}_{\varepsilon}^{*} W=\bar{\lambda}_{\varepsilon} W$ for some non-zero $W \in \mathcal{L}_{\varepsilon}$. Then, by Lemma 9

$$
\mathcal{T} \mathcal{A}_{\varepsilon} \mathcal{T} W=\bar{\lambda}_{\varepsilon} W
$$

Note that $\mathcal{T}^{2}$ is an identity operator. We apply $\mathcal{T}$ to (47) to get $\mathcal{A}_{\varepsilon} \mathcal{T} W=\bar{\lambda}_{\varepsilon} \mathcal{T} W$. Since $W \neq 0$, it follows that $\mathcal{T} W \neq 0$ is an eigenfunction of $\mathcal{A}_{\varepsilon}$ corresponding to $\bar{\lambda}_{\varepsilon}$.

Corollary 2. Let $U_{\varepsilon}$ be an eigenfunction corresponding to an eigenvalue $\lambda_{\varepsilon}$ of $\mathcal{A}_{\varepsilon}$ and let $U_{\varepsilon}^{*}$ be an eigenfunction corresponding to the same eigenvalue $\lambda_{\varepsilon}^{*}=\lambda_{\varepsilon}$ of $\mathcal{A}_{\varepsilon}^{*}$. The following statements hold.

(i) If $\lambda_{\varepsilon} \notin \mathbb{R}$, then $\left(U_{\varepsilon}, U_{\varepsilon}^{*}\right)_{\mathcal{L}_{\varepsilon}}=0$.

(ii) If $\bar{\lambda}_{\varepsilon}, U_{\varepsilon}^{*}$ is an eigenmode of $\mathcal{A}_{\varepsilon}^{*}$, then $\bar{\lambda}_{\varepsilon}, \mathcal{T} U_{\varepsilon}^{*}$ is an eigenmode of $\mathcal{A}_{\varepsilon}$.

(iii) If $\lambda_{\varepsilon}, U_{\varepsilon}$ is an eigenmode of $\mathcal{A}_{\varepsilon}$, then $\lambda_{\varepsilon}, \mathcal{T} U_{\varepsilon}$ is an eigenmode of $\mathcal{A}_{\varepsilon}^{*}$.

(iv) If $\lambda_{\varepsilon}, U_{\varepsilon}$ is an eigenmode of $\mathcal{A}_{\varepsilon}$ and $\lambda_{\varepsilon} \notin \mathbb{R}$, then $\left(U_{\varepsilon}, \mathcal{T} U_{\varepsilon}\right)_{\mathcal{L}_{\varepsilon}}=0$.

(v) Any eigenfunction $U_{\varepsilon}$ corresponding to a non-real eigenvalue satisfies the equality

$$
\int_{a}^{b} \alpha\left|\left(u_{1}^{\varepsilon}\right)^{\prime}\right|^{2} \mathrm{~d} x+\int_{a}^{b} k\left|u_{3}^{\varepsilon}\right|^{2} \mathrm{~d} x=\int_{a}^{b} \rho_{\varepsilon}\left|u_{2}^{\varepsilon}\right|^{2} \mathrm{~d} x
$$

Proof. Claim (i) is proved by noticing that for $\bar{\lambda}_{\varepsilon} \neq \lambda_{\varepsilon}^{*}$ the corresponding eigenspaces of $\mathcal{A}_{\varepsilon}$ and $\mathcal{A}_{\varepsilon}^{*}$ are orthogonal. Claim (ii) has been shown in the proof of Lemma 10. Thus $U_{\varepsilon}=\mathcal{T} U_{\varepsilon}^{*}$ is an eigenvector of $\mathcal{A}_{\varepsilon}$. Since $\mathcal{T}^{-1}=\mathcal{T}$ we obtain $U_{\varepsilon}^{*}=\mathcal{T} U_{\varepsilon}$. Note that the kernel of $\mathcal{T}$ is trivial, so $U_{\varepsilon}^{*} \neq 0$. Thus (iii) is proved. Claim (iv) follows from applying (i) to (iii). Finally, $(v)$ is a component-wise representation of (iv). 
REMARK 2. We can interpret the norm of eigenvibrations as the energy; namely,

$$
E_{\text {total }}\left(U_{\varepsilon}\right)=\left\|U_{\varepsilon}\right\|_{\mathcal{L}_{\varepsilon}}^{2}=\int_{a}^{b} \alpha\left|\left(u_{1}^{\varepsilon}\right)^{\prime}\right|^{2} \mathrm{~d} x+\int_{a}^{b} \rho_{\varepsilon}\left|u_{2}^{\varepsilon}\right|^{2} \mathrm{~d} x+\int_{a}^{b} k\left|u_{3}^{\varepsilon}\right|^{2} \mathrm{~d} x .
$$

The terms on the right-hand side are, in that order, the elastic energy $E_{\text {elastic }}$, the kinetic energy $E_{\text {kinetic }}$ and the thermal energy $E_{\text {thermal }}$. Then (48) shows that eigenvibrations with non-real eigenvalues have an equal distribution between kinetic and thermo-elastic energies:

$$
E_{\text {kinetic }}\left(U_{\varepsilon}\right)=E_{\text {thermal }}\left(U_{\varepsilon}\right)+E_{\text {elastic }}\left(U_{\varepsilon}\right)=\frac{1}{2} E_{\text {total }}\left(U_{\varepsilon}\right) .
$$

Moreover, each energetic contribution $E_{\text {kinetic }}, E_{\text {thermal }}$ and $E_{\text {elastic }}$ is non-zero.

6. The limit behaviour, $m<1$. In the case $m<1$, the limit eigenvalue problem is

$$
-u_{2}=\lambda_{0} u_{1}, \quad-\left(\alpha u_{1}^{\prime}\right)^{\prime}+\left(\beta u_{3}\right)^{\prime}=\lambda_{0} p u_{2}, \quad-\left(\varkappa u_{3}^{\prime}\right)^{\prime}+\beta u_{2}^{\prime}=\lambda_{0} k u_{3} \quad \text { in } \Omega_{0},
$$

where $\Omega_{0}=(a, 0) \cup(0, b)$, with the boundary and interface conditions

$$
u_{j}(a)=u_{j}(b)=0, \quad \llbracket u_{j} \rrbracket_{0}=\left[\left[u_{j}^{\prime}\right]\right]_{0}=0 \quad \text { for } j=1,2,3 .
$$

REMARK 3. Similarly as for problem (7)-(10), it can be proved that the spectrum of (49)-(50) is discrete. That is, it has the same properties as the spectrum of the operator $\mathcal{A}_{\varepsilon}$ described in Corollary 1. Likewise, the completeness of generalised eigenfunctions discussed at the beginning of Section 5 holds for (49)-(50) as well.

We introduce an operator $\mathcal{Q}_{0}: \mathcal{H} \rightarrow \mathcal{H}$ such that $\left(\mathcal{Q}_{0} U, V\right)_{\mathcal{H}}=(U, V)_{\mathcal{L}^{0}}$ for every $U, V \in \mathcal{H}$, where $\mathcal{L}^{0}:=H_{0}^{1}(a, b) \times L_{p}^{2}(a, b) \times L_{k}^{2}(a, b)$. Then the eigenvalue problem (49)(50) can be formulated as $\mathcal{B} U=\lambda_{0} \mathcal{Q}_{0} U$ in $\mathcal{H}$. The latter is equivalent to $\mathcal{M}_{0} U=\lambda_{0}^{-1} U$ in $\mathcal{H}$, with a bounded operator $\mathcal{M}_{0}:=\mathcal{B}^{-1} \mathcal{Q}_{0}$. Similarly, as it is shown in the previous sections, there exists a densely defined operator $\mathcal{G}_{0}: \mathcal{H} \rightarrow \mathcal{H}$ with domain $\mathfrak{D}\left(\mathcal{G}_{0}\right)=$ $\mathfrak{R}\left(\mathcal{M}_{0}\right)$ such that its inverse coincides with $\mathcal{M}_{0}, \mathcal{G}_{0}^{-1}=\mathcal{M}_{0}$. We introduce the resolvent of the operator $\mathcal{G}_{0}$, namely $\mathcal{R}\left(\zeta, \mathcal{G}_{0}\right)=\left(\mathcal{G}_{0}-\zeta\right)^{-1}$. Note that we already constructed explicitly $\mathcal{R}\left(0, \mathcal{G}_{0}\right)=\mathcal{G}_{0}^{-1}=\mathcal{M}_{0}=\mathcal{B}^{-1} \mathcal{Q}_{0}$.

We now establish the estimates between the resolvents $\mathcal{R}\left(\zeta, \mathcal{G}_{\varepsilon}\right)=\left(\mathcal{G}_{\varepsilon}-\zeta\right)^{-1}$ and $\mathcal{R}\left(\zeta, \mathcal{G}_{0}\right)$ as $\varepsilon \rightarrow 0$ for any $m<1$. Again, we already constructed explicitly $\mathcal{R}\left(0, \mathcal{G}_{\varepsilon}\right)=$ $\mathcal{G}_{\varepsilon}^{-1}=\mathcal{M}_{\varepsilon}=\mathcal{B}^{-1} \mathcal{Q}_{\varepsilon}$, which depends on $\varepsilon$ and $m$ only via the multiplier $\mathcal{Q}_{\varepsilon}$. Then the asymptotic behaviour of the resolvent as $\varepsilon \rightarrow 0$ is determined by the asymptotic behaviour of the operator $\mathcal{Q}_{\varepsilon}$.

LEMmA 11. If $m<1$, then for small $\varepsilon$ we have $\left\|\mathcal{R}\left(\zeta, \mathcal{G}_{\varepsilon}\right)-\mathcal{R}\left(\zeta, \mathcal{G}_{0}\right)\right\| \leq C \varepsilon^{\gamma}$ with $\gamma:=\min \{1,1-m\}$ and a positive constant $C$ independent of $\varepsilon$ and $m$.

Proof. By virtue of [6, IV.3.13],

$$
\begin{aligned}
\left\|\mathcal{R}\left(\zeta, \mathcal{G}_{\varepsilon}\right)-\mathcal{R}\left(\zeta, \mathcal{G}_{0}\right)\right\| & \leq C\left\|\mathcal{R}\left(0, \mathcal{G}_{\varepsilon}\right)-\mathcal{R}\left(0, \mathcal{G}_{0}\right)\right\|=C\left\|\mathcal{M}_{\varepsilon}-\mathcal{M}_{0}\right\| \\
& =C\left\|\mathcal{B}^{-1}\left(\mathcal{Q}_{\varepsilon}-\mathcal{Q}_{0}\right)\right\| \leq C\left\|\mathcal{B}^{-1}\right\|\left\|\mathcal{Q}_{\varepsilon}-\mathcal{Q}_{0}\right\| \\
& \leq C\left\|\mathcal{Q}_{\varepsilon}-\mathcal{Q}_{0}\right\| .
\end{aligned}
$$


For arbitrary elements $U, V \in \mathcal{H}$ we consider

$$
\begin{aligned}
\left(\left(\mathcal{Q}_{\varepsilon}-\mathcal{Q}_{0}\right) U, V\right)_{\mathcal{H}} & =\left(\mathcal{Q}_{\varepsilon} U, V\right)_{\mathcal{H}}-\left(\mathcal{Q}_{0} U, V\right)_{\mathcal{H}}=(U, V)_{\mathcal{L}_{\varepsilon}}-(U, V)_{\mathcal{L}^{0}} \\
& =\int_{a}^{b}\left(\rho_{\varepsilon}-p\right) u_{2} v_{2} \mathrm{~d} x=\int_{-\varepsilon}^{\varepsilon}\left[\varepsilon^{-m} q\left(\frac{x}{\varepsilon}\right)-p(x)\right] u_{2} v_{2} \mathrm{~d} x
\end{aligned}
$$

Then by Lemma 13 in the Appendix, $\left|\left(\left(\mathcal{Q}_{\varepsilon}-\mathcal{Q}_{0}\right) U, V\right)_{\mathcal{H}}\right| \leq C \varepsilon^{\gamma}\left\|u_{2}\right\|_{H_{0}^{1}(a, b)}\left\|v_{2}\right\|_{H_{0}^{1}(a, b)}$ $\leq C \varepsilon^{\gamma}\|U\|_{\mathcal{H}}\|V\|_{\mathcal{H}}$ for any $U, V \in \mathcal{H}$. Hence, $\left\|\mathcal{Q}_{\varepsilon}-\mathcal{Q}_{0}\right\| \leq C \varepsilon^{\gamma}$. A substitution into (51) proves the claim.

Theorem 1. Let $m<1$. Then the eigenvalues $\lambda_{\varepsilon}$ of problem (7)-(10) converge to the eigenvalues $\lambda_{0}$ of problem (49)-(50) saving multiplicity. Let $\mathcal{P}_{\varepsilon}$ be the eigenprojector to the generalised eigenspace of $\mathcal{M}_{\varepsilon}$ corresponding to $\lambda_{\varepsilon}$, and let $\mathcal{P}_{0}$ be the eigenprojector to the generalised eigenspace of $\mathcal{M}_{0}$ corresponding to $\lambda_{0}$. Then

$$
\left\|\mathcal{P}_{\varepsilon}-\mathcal{P}_{0}\right\| \leq C \varepsilon^{\gamma} \quad \text { with } \gamma=\min \{1,1-m\} .
$$

Moreover, for each eigenvalue $\lambda_{0}$ of (49)-(50), there is a sequence of eigenvalues $\left\{\lambda_{\varepsilon}\right\}_{\varepsilon \rightarrow 0}$ of (7)-(10) such that $\lambda_{\varepsilon} \rightarrow \lambda_{0}$ and such that estimate (52) holds.

Proof. Lemma 11 states the resolvent convergence $\mathcal{R}\left(\cdot, \mathcal{G}_{\varepsilon}\right) \rightarrow \mathcal{R}\left(\cdot, \mathcal{G}_{0}\right)$ in norm as $\varepsilon \rightarrow 0$. Then the eigenvalue convergence saving multiplicity follows [2, p. 35]. Further, each eigenvalue $\lambda_{0}$ can be encircled with a contour $\Gamma$ such that no other eigenvalue of $\mathcal{G}_{0}$ is inside or on the contour. Then the eigenprojector to the generalised eigenspace corresponding to $\lambda_{0}$ is given by the Riesz projector

$$
\mathcal{P}_{0}=\frac{1}{2 \pi i} \int_{\Gamma} \mathcal{R}\left(\zeta, \mathcal{G}_{0}\right) \mathrm{d} \zeta .
$$

Since $\lambda_{\varepsilon} \rightarrow \lambda_{0}$, for $\varepsilon$ small enough, $\lambda_{\varepsilon}$ is strictly inside $\Gamma$. Then again the eigenprojector corresponding to $\lambda_{\varepsilon}$ is given by

$$
\mathcal{P}_{\varepsilon}=\frac{1}{2 \pi i} \int_{\Gamma} \mathcal{R}\left(\zeta, \mathcal{G}_{\varepsilon}\right) \mathrm{d} \zeta .
$$

Since the contour $\Gamma$ does not depend on $\varepsilon$ and consists only of regular points of $\mathcal{G}_{\varepsilon}$ and $\mathcal{G}_{0}$, we can estimate

$$
\left\|\mathcal{P}_{\varepsilon}-\mathcal{P}_{0}\right\| \leq C_{1} \int_{\Gamma}\left\|\mathcal{R}\left(\zeta, \mathcal{G}_{\varepsilon}\right)-\mathcal{R}\left(\zeta, \mathcal{G}_{0}\right)\right\| \mathrm{d} \zeta \leq C_{2} \varepsilon^{\gamma}
$$

by virtue of Lemma 11 .

If we assume that there exists $\lambda_{0}$ which cannot be approached by a sequence $\left\{\lambda_{\varepsilon}\right\}$, then there are no $\lambda_{\varepsilon}$ in a suitable neighbourhood $N$ of $\lambda_{0}$. Choose a contour $\Gamma_{1}$ inside $N$ encircling $\lambda_{0}$. Then the corresponding Riesz projectors to the linear subspaces, whose eigenvalues are inside $\Gamma_{1}$, are given by (53) and (54) with $\Gamma=\Gamma_{1}$. Therefore the projectors satisfy (55). Hence, for small $\varepsilon$ we obtain $\operatorname{dim} \mathcal{P}_{\varepsilon}=\operatorname{dim} \mathcal{P}_{0}$. This contradicts the assumption that inside $\Gamma_{1}$ there are no eigenvalues $\lambda_{\varepsilon}$ of $\mathcal{G}_{\varepsilon}$. 
7. The limit behaviour, $m=1$. For $m=1$ the limit eigenvalue problem is

$$
\begin{gathered}
-u_{2}=\lambda_{0} u_{1}, \quad-\left(\alpha u_{1}^{\prime}\right)^{\prime}+\left(\beta u_{3}\right)^{\prime}=\lambda_{0} p u_{2}, \quad-\left(\varkappa u_{3}^{\prime}\right)^{\prime}+\beta u_{2}^{\prime}=\lambda_{0} k u_{3} \quad \text { in } \Omega_{0}, \\
u_{j}(a)=u_{j}(b)=0, \quad \llbracket u_{j} \rrbracket_{0}=0, \quad j=1,2,3, \\
\llbracket u_{1}^{\prime} \rrbracket_{0}=-\lambda_{0} q_{0} \alpha(0)^{-1} u_{2}(0), \quad \llbracket u_{2}^{\prime} \rrbracket_{0}=-\lambda_{0} \llbracket u_{1}^{\prime} \rrbracket_{0}, \quad \llbracket u_{3}^{\prime} \rrbracket_{0}=0
\end{gathered}
$$

with

$$
q_{0}=\int_{-1}^{1} q(\xi) \mathrm{d} \xi
$$

REmark 4. Similarly as for problem (7)-(10), it can be proved that the spectrum of (56) -(58) is discrete. That is, it has the same properties as the spectrum of the operator $\mathcal{A}_{\varepsilon}$ described in Corollary 1 .

Note that the variational formulation of the problem leads to

$$
\tau(U, \Phi)+\alpha(0) \bar{\varphi}_{2}(0) \llbracket u_{1}^{\prime} \rrbracket_{0}=\lambda_{0}(U, \Phi)_{\mathcal{L}^{0}} \quad \text { for every } \Phi \in \mathcal{H} .
$$

The latter along with the transmission conditions (58) implies

$$
\tau(U, \Phi)=\lambda_{0}\left((U, \Phi)_{\mathcal{L}^{0}}+q_{0} u_{2}(0) \bar{\varphi}_{2}(0)\right) \quad \text { for every } \Phi \in \mathcal{H} .
$$

We introduce a bounded operator $\mathcal{Q}_{1}: \mathcal{H} \rightarrow \mathcal{H}$ such that

$$
\left(\mathcal{Q}_{1} U, V\right)_{\mathcal{H}}=(U, V)_{\mathcal{L}^{0}}+q_{0} u_{2}(0) \bar{v}_{2}(0) \quad \text { for every } U, V \in \mathcal{H} .
$$

Then eigenvalue problem (56) $-(58)$ can be formulated as $\mathcal{B} U=\lambda_{0} \mathcal{Q}_{1} U$ in $\mathcal{H}$. The latter is equivalent to $\mathcal{M}_{1} U=\lambda_{0}^{-1} U$ in $\mathcal{H}$, with a bounded operator $\mathcal{M}_{1}:=\mathcal{B}^{-1} \mathcal{Q}_{1}$. Similarly, as is shown in the previous sections, it can be shown that there exists a densely defined operator $\mathcal{G}_{1}: \mathcal{H} \rightarrow \mathcal{H}$ with domain $\mathfrak{D}\left(\mathcal{G}_{1}\right)=\mathfrak{R}\left(\mathcal{M}_{1}\right)$ such that its inverse coincides with $\mathcal{M}_{1}, \mathcal{G}_{1}^{-1}=\mathcal{M}_{1}$. We introduce the resolvent

$$
\mathcal{R}\left(\zeta, \mathcal{G}_{1}\right)=\left(\mathcal{G}_{1}-\zeta\right)^{-1} .
$$

Note that we already constructed explicitly $\mathcal{R}\left(0, \mathcal{G}_{1}\right)=\mathcal{G}_{1}^{-1}=\mathcal{M}_{1}=\mathcal{B}^{-1} \mathcal{Q}_{1}$.

Lemma 12. If $m=1$, then for small $\varepsilon$ we have $\left\|\mathcal{R}\left(\zeta, \mathcal{G}_{\varepsilon}\right)-\mathcal{R}\left(\zeta, \mathcal{G}_{1}\right)\right\| \leq C \varepsilon^{1 / 2}$ with $C>0$ independent of $\varepsilon$.

Proof. Similarly to the proof of Lemma 11, we establish

$$
\left\|\mathcal{R}\left(\zeta, \mathcal{G}_{\varepsilon}\right)-\mathcal{R}\left(\zeta, \mathcal{G}_{1}\right)\right\| \leq C\left\|\mathcal{Q}_{\varepsilon}-\mathcal{Q}_{1}\right\| .
$$

For arbitrary elements $U, V \in \mathcal{H}$ we consider

$$
\begin{aligned}
\left(\left(\mathcal{Q}_{\varepsilon}-\mathcal{Q}_{1}\right) U, V\right)_{\mathcal{H}} & =(U, V)_{\mathcal{L}_{\varepsilon}}-(U, V)_{\mathcal{L}^{0}}-q_{0} u_{2}(0) v_{2}(0) \\
& =\varepsilon^{-1} \int_{-\varepsilon}^{\varepsilon} q\left(\frac{x}{\varepsilon}\right) u_{2} v_{2} \mathrm{~d} x-\int_{-\varepsilon}^{\varepsilon} p(x) u_{2} v_{2} \mathrm{~d} x-q_{0} u_{2}(0) v_{2}(0) .
\end{aligned}
$$

Then by Lemma 13 in the Appendix, we obtain

$$
\left\|\left(\left(\mathcal{Q}_{\varepsilon}-\mathcal{Q}_{1}\right) U, V\right)_{\mathcal{H}}\right\| \leq C \varepsilon^{\frac{1}{2}}\left\|u_{2}\right\|_{H_{0}^{1}(a, b)}\left\|v_{2}\right\|_{H_{0}^{1}(a, b)} \leq C \varepsilon^{\frac{1}{2}}\|U\|_{\mathcal{H}}\|V\|_{\mathcal{H}}
$$

for any $U, V \in \mathcal{H}$. Hence, $\left\|\mathcal{Q}_{\varepsilon}-\mathcal{Q}_{1}\right\| \leq C \varepsilon^{1 / 2}$. A substitution in (59) proves the claim. 
Theorem 2. Let $m=1$. Then the eigenvalues $\lambda_{\varepsilon}$ of (7)-(10) converge to the eigenvalues $\lambda_{0}$ of (56) saving multiplicity. Let $\mathcal{P}_{\varepsilon}$ be the eigenprojector to the generalised eigenspace corresponding to $\lambda_{\varepsilon}$, and let $\mathcal{P}_{1}$ be the eigenprojector to the generalised eigenspace corresponding to $\lambda_{0}$. Then the following estimate holds:

$$
\left\|\mathcal{P}_{\varepsilon}-\mathcal{P}_{1}\right\| \leq C \varepsilon^{\frac{1}{2}} .
$$

Moreover, for each eigenvalue $\lambda_{0}$ of (56) -(58) there is a sequence of eigenvalues $\left\{\lambda_{\varepsilon}\right\}_{\varepsilon \rightarrow 0}$ of (7)-(10) such that $\lambda_{\varepsilon} \rightarrow \lambda_{0}$ and such that estimate (60) holds.

The idea of the proof is the same as that for Theorem 1 and is thus omitted; it relies on the estimates of Lemma 12, We remark that for $m=1$, the localised mass concentration influences the limit eigenfrequencies and eigenvibrations represented in transmission conditions (58) involving momentum of mass $q_{0}$ and the spectral parameter (see [4] for the same effect in the isothermal elastic problem).

Discussion. We want to finish with a brief comparison of the limit behaviour in the cases $m<1$ and $m=1$. Theorem 1 shows that there is no influence of localised perturbation in the mass density $\varepsilon^{-m} q$ on the limit eigenfrequencies and the generalised eigenspaces for $m<1$. This makes the expectation sufficiently rigorous that for $m<1$ the added mass is small enough to be neglected since

$$
\int_{-\varepsilon}^{\varepsilon} \varepsilon^{-m} q\left(\frac{x}{\varepsilon}\right) \mathrm{d} x=\varepsilon^{1-m} \int_{-1}^{1} q(\xi) \mathrm{d} \xi \rightarrow 0 \quad \text { as } \varepsilon \rightarrow 0 .
$$

In contrast, Theorem 2 shows for $m=1$ a dependence of the eigenvibrations on the concentrated mass $q_{0}$; see (58). This makes the intuition profound that larger values of $m$ have a stronger impact on the limit behaviour, leading to localisation of eigenvibrations; this has so far been proved rigorously in the isothermal case only [4].

\section{Appendix.}

Lemma 13 (Golovaty, Nazarov, Oleinik, Soboleva 4]). Let $p \in C[a, b], q \in C[-1,1]$ and $q_{0}:=\int_{-1}^{1} q(\xi) \mathrm{d} \xi$. For any $u, v \in H_{0}^{1}(a, b)$ the following estimates hold:

$$
\begin{aligned}
\left\|\varepsilon^{-1} \int_{-\varepsilon}^{\varepsilon} q\left(\frac{x}{\varepsilon}\right) u(x) v(x) \mathrm{d} x-q_{0} u(0) v(0)\right\| & \leq C \varepsilon^{\frac{1}{2}}\|u\|_{H_{0}^{1}(a, b)}\|v\|_{H_{0}^{1}(a, b)}, \\
\left|\int_{-\varepsilon}^{\varepsilon} p(x) u(x) v(x) \mathrm{d} x\right| & \leq C \varepsilon\|u\|_{H_{0}^{1}(a, b)}\|v\|_{H_{0}^{1}(a, b)} .
\end{aligned}
$$

For the reader's convenience, we give the proof here.

Proof. The second claim follows from the compact embedding $C(a, b) \subset H_{0}^{1}(a, b)$, since

$$
\begin{aligned}
\left|\int_{-\varepsilon}^{\varepsilon} p(x) u(x) v(x) \mathrm{d} x\right| & \leq \int_{-\varepsilon}^{\varepsilon}|p(x) u(x) v(x)| \mathrm{d} x \\
& \leq 2 \varepsilon \max _{x \in[a, b]}|p(x) u(x) v(x)| \leq C \varepsilon\|u\|_{H_{0}^{1}(a, b)}\|v\|_{H_{0}^{1}(a, b)} .
\end{aligned}
$$


To prove the first claim, we note that

$$
\begin{aligned}
& \left|\varepsilon^{-1} \int_{-\varepsilon}^{\varepsilon} q\left(\frac{x}{\varepsilon}\right) u(x) v(x) \mathrm{d} x-q_{0} u(0) v(0)\right|=\left|\int_{-1}^{1} q(\xi)(u(\varepsilon \xi) v(\varepsilon \xi)-u(0) v(0)) \mathrm{d} \xi\right| \\
& =\left|\int_{-1}^{1} q(\xi)\left(\int_{0}^{\varepsilon \xi}\left(u^{\prime} v-u v^{\prime}\right) \mathrm{d} t\right) \mathrm{d} \xi\right| \\
& \leq \int_{-1}^{1} q(\xi)\left|\int_{0}^{\varepsilon \xi} u^{\prime} v \mathrm{~d} t\right| \mathrm{d} \xi+\int_{-1}^{1} q(\xi)\left|\int_{0}^{\varepsilon \xi} u v^{\prime} \mathrm{d} t\right| \mathrm{d} \xi .
\end{aligned}
$$

For any $\xi \in(-1,1)$, we have

$$
\begin{aligned}
\left|\int_{0}^{\varepsilon \xi} u^{\prime} v \mathrm{~d} t\right| & \leq\left|\int_{0}^{\varepsilon \xi}\left(u^{\prime}\right)^{2} \mathrm{~d} t\right|^{\frac{1}{2}}\left|\int_{0}^{\varepsilon \xi} v^{2} \mathrm{~d} t\right|^{\frac{1}{2}} \\
& \leq\left|\int_{a}^{b}\left(u^{\prime}\right)^{2} \mathrm{~d} t\right|^{\frac{1}{2}}|\varepsilon \xi|^{\frac{1}{2}} \max _{t \in[a, b]}\left|v^{2}(t)\right| \leq C|\varepsilon \xi|^{\frac{1}{2}}\|u\|_{H_{0}^{1}(a, b)}\|v\|_{H_{0}^{1}(a, b)}
\end{aligned}
$$

Therefore

$$
\int_{-1}^{1} q(\xi)\left|\int_{0}^{\varepsilon \xi} u^{\prime} v \mathrm{~d} t\right| \mathrm{d} \xi \leq C \varepsilon^{\frac{1}{2}}\|u\|_{H_{0}^{1}(a, b)}\|v\|_{H_{0}^{1}(a, b)} .
$$

Since the last estimate is symmetric in $u$ and $v$, the other term in (63) satisfies the same estimate; this proves (61).

Acknowledgements. The authors are grateful to V. P. Smyshlyaev and I. V. Kamotski for helpful discussions. Both authors gratefully acknowledge the financial support of the EPSRC (GR / S99037 / 1 and GR / S99044 / 1).

\section{REFERENCES}

[1] Christer Bennewitz and Yoshimi Saitō. Approximation numbers of Sobolev embedding operators on an interval. J. London Math. Soc. (2), 70(1):244-260, 2004. MR2064761 (2005c:46037)

[2] I. C. Gohberg and M. G. Krĕ̌n. Vvedenie v teoriyu lineinykh nesamosopryazhennykh operatorov $v$ gilbertovom prostranstve. Izdat. "Nauka", Moscow, 1965. MR0220070 (36:3137)

[3] Yu. D. Golovaty. Spectral properties of oscillatory systems with added masses. Trudy Moskov. Mat. Obshch., 54:29-72, 278, 1992; translation in Trans. Moscow Math. Soc., 1993:23-59, 1993. MR.1256922 (95b:73012)

[4] Yu. D. Golovaty, S. A. Nazarov, O. A. Oleinik, and T. S. Soboleva. Natural oscillations of a string with an additional mass. Sibirsk. Mat. Zh., 29(5):71-91, 237, 1988. MR971229 (90e:34044)

[5] Song Jiang and Reinhard Racke. Evolution equations in thermoelasticity, volume 112 of Chapman 85 Hall/CRC Monographs and Surveys in Pure and Applied Mathematics. Chapman \& Hall/CRC, Boca Raton, FL, 2000. MR:1774100 (2001g:74013)

[6] Tosio Kato. Perturbation theory for linear operators. Classics in Mathematics. Springer-Verlag, Berlin, 1995. Reprint of the 1980 edition. MR1335452 (96a:47025)

[7] O. A. Oleinik, J. Sanchez-Hubert, and G. A. Yosifian. On vibrations of a membrane with concentrated masses. Bull. Sci. Math., 115(1):1-27, 1991. MR.1086936 (92a:73021)

[8] J. Sanchez Hubert and E. Sánchez-Palencia. Vibration and coupling of continuous systems. SpringerVerlag, Berlin, 1989. Asymptotic methods. MR996423 (91c:00018)

[9] E. Sánchez-Palencia. Perturbation of eigenvalues in thermoelasticity and vibration of systems with concentrated masses. In Trends and applications of pure mathematics to mechanics (Palaiseau, 1983), volume 195 of Lecture Notes in Phys., pages 346-368. Springer, Berlin, 1984. MR755735 (85m:73010) 
[10] E. Sánchez-Palencia and H. Tchatat. Vibration de systèmes élastiques avec des masses concentrées. Rend. Sem. Mat. Univ. Politec. Torino, 42(3):43-63, 1984. MR834781(87i:73039)

[11] Enrique Sánchez-Palencia. Nonhomogeneous media and vibration theory, volume 127 of Lecture Notes in Physics. Springer-Verlag, Berlin, 1980. MR.578345 (82j:35010)

[12] Yakov Yakubov. Completeness of root functions and elementary solutions of the thermoelasticity system. Math. Models Methods Appl. Sci., 5(5):587-598, 1995. MR1347149 (96c:35137) 